\title{
A 4-COLOR THEOREM FOR TOROIDAL GRAPHS
}

\author{
HUDSON V. KRONK ${ }^{1}$ AND ARTHUR T. WHITE ${ }^{2}$
}

\begin{abstract}
It is well known that any graph imbedded in the torus has chromatic number at most seven, and that seven is attained by the graph $K_{7}$. In this note we show that any toroidal graph containing no triangles has chromatic number at most four, and produce an example attaining this upper bound. The results are then extended for arbitrary girth.
\end{abstract}

1. Introduction. The chromatic number of a graph $G$, denoted by $\chi(G)$, is the minimum number of colors that can be assigned to the points of $G$ so that adjacent points are colored differently. The Four Color Conjecture is that any planar graph has chromatic number at most four; the Five Color Theorem states that $\chi(G) \leqq 5$, if $G$ is planar. Grötzsch [2] has shown that a planar graph having no triangles has chromatic number at most three; the cycle $C_{5}$ shows that this bound cannot be improved. It is well known that any toroidal graph has chromatic number at most seven, and that this bound is attained by the complete graph $K_{7}$. In this note we find an upper bound for the chromatic number of toroidal graphs having no triangles, and show that this bound is best possible. We also consider toroidal graphs of arbitrary girth.

A connected graph $G$ is said to be $n$-line-critical if $\chi(G)=n$ but, for any line $e$ of $G, \chi(G-e)=n-1$. We will use the following theorem of Dirac [1]; $p$ and $q$ denote the number of points and lines of $G$, respectively.

THEOREM 1. If $G$ is an $n$-line-critical graph, $n \geqq 4$, and if $G \neq K_{n}$, then $2 q \geqq(n-1) p^{\prime}+n-3$.

We will also use the following well-known consequence of the generalized euler polyhedral formula (see, for example, [3, p. 118]):

THEOREM 2. If $G$ is a connected graph of genus $\gamma$ and having no triangles, then $\gamma \geqq 1-p / 2+q / 4$.

Received by the editors August 26, 1971.

AMS 1970 subject classifications. Primary 05C10, 05C15; Secondary 55A15.

Key words and phrases. Graph, chromatic number, torus, girth.

${ }^{1}$ Research supported by SUNY Faculty Research Fellowship.

${ }^{2}$ Research supported in part by Western Michigan University (Faculty Research Fellowship).

(c) American Mathematical Society 1972 
2. The main result. We are now prepared to extend Grötzsch's theorem to the torus.

THEOREM 3. If a graph $G$ having no triangles is imbedded in the torus, then $\chi(G) \leqq 4$.

Proof. Let $\chi(G)=n \geqq 5$. We first assume that $G$ is $n$-line-critical, and hence connected. Since $G$ has no triangles, $G \neq K_{n}$. By Theorem 1 ,

$$
2 q \geqq(n-1) p+n-3 .
$$

Now if $G$ has genus one, then by Theorem 2,

$$
4 p \geqq 2 q \text {. }
$$

Combining (1) and (2), we see that $n \leqq 4$. If $G$ has genus zero, then $n \leqq 3$, by Grötzsch's theorem. In either case we have a contradiction, so that $n \leqq 4$.

Now suppose that $G$ is not $n$-line-critical. Then $G$ contains an $n$-linecritical subgraph $H$, and the argument above shows that $\chi(G)=\chi(H)=$ $n \leqq 4$.

The graph of Figure 1, constructed by Mycielski [4] as an example of a graph having no triangles and chromatic number four, also has genus one, so that the bound of Theorem 3 cannot be improved.

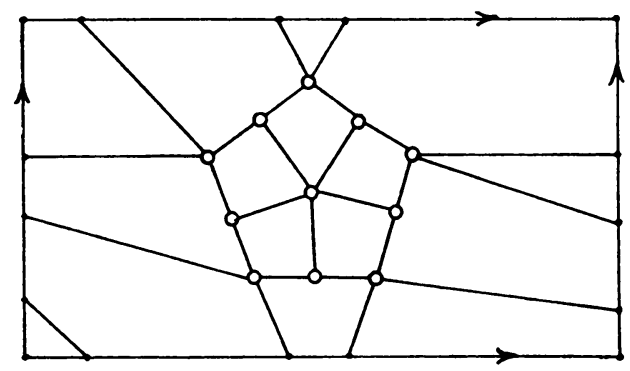

FIGURE 1

3. Extensions for arbitrary girth. The girth $g(G)$ of a graph $G$ is the length of a shortest cycle (if any) in $G$. A graph $G$ with cycles but no triangles has $g(G) \geqq 4$; the graph of Figure 1 has girth exactly four.

If $g(G) \geqq 6$, then each region in an imbedding for $G$ has at least six edges in its boundary, so that $2 q \geqq 6 r$, where $r$ is the total number of regions. As before, we may assume that $\gamma(G)=1$ and that $G$ is $n$-line-critical. If $n \geqq 4$, then $2 q \geqq 3 p+1$, by Theorem 1 . By the euler formula for the torus, 
then:

$$
0=p-q+r \leqq \frac{2 q-1}{3}-q+\frac{q}{3}=-\frac{1}{3},
$$

an obvious contradiction. Hence toroidal graphs of girth six or more have chromatic number three or less. This bound is also best possible, as an appropriate subdivision of the Petersen graph (shown imbedded in the torus in Figure 2) can always be found, having girth $m(m \geqq 5)$, genus one, and chromatic number three.

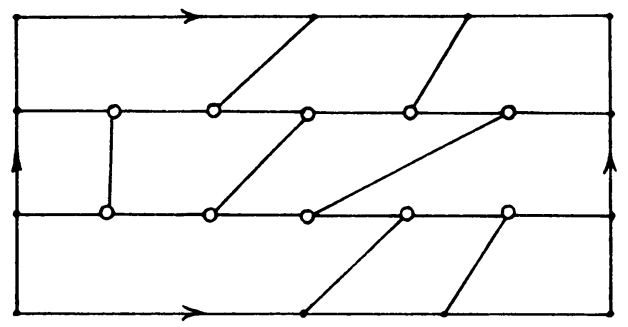

FIGURE 2

For $g(G)=5$ and $\gamma(G)=1$, it follows from Theorem 3 that $\chi(G) \leqq 4$. This bound can also be established by employing the following result of Szekeres and Wilf [5]:

THEOREM 4. For any graph $G, \chi(G) \leq 1+\max \delta\left(G^{\prime}\right)$, where the maximum is taken over all induced subgraphs $G^{\prime}$ of $G$, and $\delta\left(G^{\prime}\right)$ denotes the minimum degree of $G^{\prime}$.

For toroidal graphs $G^{\prime}$ having girth 5 , if $\delta\left(G^{\prime}\right) \geqq 4$, then $2 q \geqq 4 p$ and also $2 q \geqq 5 r$, so that

$$
0=p-q+r \leqq \frac{q}{2}-q+\frac{2}{5} q=-\frac{q}{10} ;
$$

hence $\delta\left(G^{\prime}\right) \leqq 3$. It now follows from Theorem 4 that $\chi(G) \leqq 4$. Whether this upper bound can be attained remains an open question.

We summarize our results in:

TheOREM 5. Let $G$ be imbedded in the torus, with $g(G)=m$. Then

$$
\begin{aligned}
\chi(G) & \leqq 7, \quad \text { if } m=3, \\
& \leqq 4, \quad \text { if } m=4 \text { or } 5, \\
& \leqq 3, \quad \text { if } m \geqq 6 .
\end{aligned}
$$

Furthermore, all the bounds are sharp, except possibly for $m=5$. 


\section{REFERENCES}

1. G. A. Dirac, A theorem of $R$. L. Brooks and a conjecture of H. Hadwiger, Proc. London Math. Soc. (3) 7 (1957), 161-195. MR 19, 161.

2. H. Grötzsch, Zur Theorie der diskreten Gebilde. VII. Ein Dreifarbensatz für dreikreisfreie Netze auf der Kugel, Wiss. Z. Martin-Luther-Univ. Halle-Wittenberg. Math. Nat. Reihe 8 (1958/59), 109-120. MR 22 \#7113c.

3. F. Harary, Graph theory, Addison-Wesley, Reading, Mass., 1969. MR 41 \#1566.

4. J. Mycielski, Sur le coloriage des graphs, Colloq. Math. 3 (1955), 161-162. MR 16, 1044.

5. G. Szekeres and H. S. Wilf, An inequality for the chromatic number of a graph, J. Combinatorial Theory 4 (1968), 1-3. MR 36 \#1356.

Department of Mathematics, State University of New York at Binghamton, Binghamton, New York 13901

Department of Mathematics, Western Michigan University, Kalamazoo, MiCHIGAN 49001 